\title{
Impact du réchauffement climatique sur l'activité avalancheuse et multiplication des avalanches humides dans les Alpes françaises
}

\author{
Mohamed NAAIM ${ }^{1}$, Nicolas ECKERT ${ }^{1}$, Gerald GIRAUD ${ }^{2}$, Thierry FAUG ${ }^{1}$, Guillaume CHAMBON ${ }^{1}$, \\ Florence NAAIM-BOUVET ${ }^{1}$, Didier RICHARD ${ }^{1}$ \\ ${ }^{1}$ Université Grenoble Alpes, Irstea, UR ETGR, 2 Rue de la papeterie, Domaine universitaire, 38402 Saint Martin d'Hères - \\ e-mail: mohamed.naaim@irstea.fr \\ ${ }^{2}$ Metéo-France-CNRM, CNRM-GAME UMR 3589, Centre d'Etudes de la Neige, 1441 rue de la Piscine, 38402 Saint Martin d'Hères.
}

\begin{abstract}
RÉSUMÉ. - Sur l'ensemble des Alpes françaises, le croisement de deux bases de données bien documentées, la chronique d'avalanches EPA et les réanalyses nivo-météorologique, a permis de quantifier la réponse de l'activité avalancheuse aux évolutions récentes du climat hivernal. Même si le signal est affecté d'une variabilité interannuelle importante, il est démontré qu'en moyenne sur les trente dernières années on observe une augmentation des altitudes d'arrêt et une diminution du nombre d'avalanche avec aérosols et du nombre moyen d'avalanches. Sur le massif du Mont-Blanc, l'article présente les résultats obtenus sur l'augmentation, quasi concomitante avec le récent réchauffement, de la proportion des avalanches humides, de même que les corrélations obtenues entre les paramètres de frottement des avalanches et les propriétés de la neige. Par la suite l'article synthétise les nouvelles avancées en matière de pression d'impact des avalanches et souligne les conséquences attendues notamment pour les avalanches humides. Enfin, l'article se conclut par la présentation synthétique des projections au prochain siècle des quantités de précipitations, de la qualité de la neige et des décalages attendus, à la fois en saisons et en altitude, de l'activité avalancheuse.
\end{abstract}

Mots clefs : Avalanches, neige humide, réchauffement climatique, impacts, aléa, risque.

\section{Impact of climate warming on avalanche activity in French Alps and increase of proportion of wet snow avalanches}

\begin{abstract}
The cross-analysis of two well documented databases, EPA snow avalanche chronicles and the reanalysis of snow mantle, allows quantifying the changes in avalanche activity as a response to climate warming. In the French Alps and even if the signal is affected by a significant variability, it is shown that, on average over the past thirty years, the run-out altitudes increased and the frequency and the proportion of powder avalanches decreased. In Chamonix valley a substantial increase of the proportion of wet snow avalanches since the seventies has been shown, concomitant to the local climate warming. The reanalysis of a large number of avalanches that occurred in Chamonix valley between 1958 and 2010 allowed drawing up significant correlations between the basal friction coefficient and the snow properties. On average, the friction coefficient increases with snow temperature. This trend is coherent with the run-out altitude increase observed over the French Alps since 1980. However the friction coefficient drops to small values when snow water content exceeds a certain threshold, hence very wet snow avalanches have large extensions. In addition, because their density and height are high and the velocity low, the pressure they develop is higher than expected notably in the run-out zone. The expected consequences on the avalanche hazard of these proved trends is presented together with the projections concerning amount and quality of snow for the next century as well as expected altitudinal and seasonal shifts in avalanche activity.
\end{abstract}

Key words: Avalanches, wet snow, climate warming, impacts, natural hazard.

\section{INTRODUCTION}

$\mathrm{Au}$ cours des récents hivers les Alpes françaises ont connu une succession de périodes répétées de forte activité d'avalanches de neige humide. Des conséquences spectaculaires en matière de dommages aux infrastructures ont été observées. Parmi les événements les plus remarquables, la destruction de la gare du télésiège de Saint-François-Longchamp (Savoie) le 2/3/2012 par une avalanche de neige humide a été marquante d'une part par la surprise occasionnée et d'autre part par la dynamique particulière de l'avalanche. Le processus qui a conduit au déclenchement de toute l'épaisseur du manteau neigeux a été très lent (la fracture au sommet de la zone de départ a évolué pendant pratiquement un mois), rendant la prévision de l'événement très difficile. L'avalanche s'est propagée à vitesse faible (moins de $1 \mathrm{~m} / \mathrm{s}$ ) et avec une forte épaisseur ; au niveau du télésiège l'épaisseur a ainsi avoisiné les $5 \mathrm{~m}$ (Figure 1).

La répétition de tels phénomènes a conduit les nivologues à s'interroger sur leurs liens avec le changement climatique, ainsi que sur les évolutions déjà avérées et celles attendues dans un avenir plus ou moins proche. Au-delà, la réflexion porte sur la prise en compte de cette évolution pour adapter nos stratégies de prévention et de protection vis-à-vis du risque avalancheux. L'objet de cet article est de faire la synthèse des connaissances, à destination des scientifiques et des ingénieurs en charge de la conception et de la mise en œuvre de la politique publique, d'une part sur les évolutions 


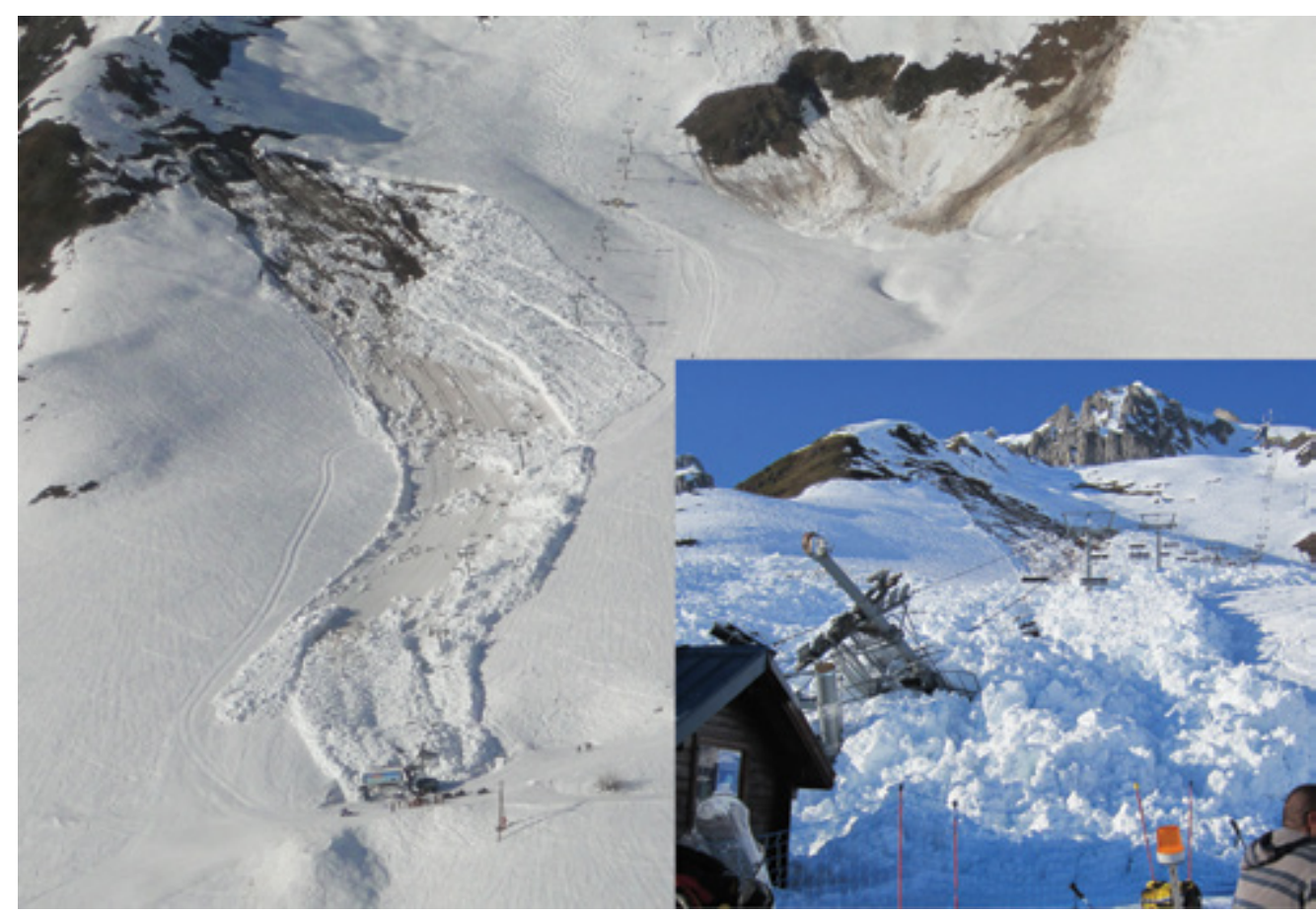

Figure 1 : Extension de l'avalanche du 2 Mars 2012 à Saint François Longchamp (Photos DAG Modane / data-avalanche.org).

avérées et attendues en matière de nombre, de type d'avalanches et d'altitude d'arrêt, et d'autre part, sur les conséquences en termes de dynamique et de pression d'impact dans les zones atteintes. Il complétera ainsi le travail de synthèse présenté par [Eckert, 2011].

Cet article commence par présenter les connaissances récentes concernant la réponse de l'activité avalancheuse du massif alpin français aux évolutions du climat hivernal. Les résultats concernent la fréquence moyenne annuelle, le type d'avalanches, à savoir sèche, humide, dense ou aérosol, et les grandeurs caractéristiques telles que la distance d'arrêt et la pression d'impact. Il se poursuit par l'analyse des conséquences attendues de ces évolutions avérées. Il se termine par la présentation synthétique des projections au prochain siècle des quantités de précipitations, de la qualité de la neige et des décalages attendus, à la fois en saisons et en altitude, de l'activité avalancheuse.

\section{REPONSE DE L'ACTIVITÉ AVALANCHEUSE AUX ÉVOLUTIONS DU CLIMAT DANS LES ALPES FRANÇAISES}

La tendance nette au réchauffement du climat de nos montagnes est désormais bien établie (Figure 2, dans [Durand et al., 2009a\&b]). L'une des conséquences les plus directes est la diminution déjà observable sur la période récente de l'enneigement à basse et moyenne altitude en termes de cumul et de durée. Mais l'on constate aussi une augmentation de la variabilité, notamment des températures hivernales à haute altitude [Beniston, 2005].

L'activité avalancheuse naturelle étant directement contrôlée par la quantité et la qualité de la neige disponible, elle est susceptible d'évoluer significativement avec les modifications du climat. Néanmoins, la réponse de l'activité avalancheuse au changement climatique est complexe. Il existe différents types d'avalanches en fonction de leur régime d'écoulement (dense/aérosol) ou du type de neige (sèche/humide). De plus, différentes variables peuvent être étudiées (nombre d'événements, distances parcourues, etc.), ainsi que plusieurs échelles de temps : hiver entier, important pour l'urbanisme, ou cycles d'activité intense de quelques jours, plus pertinents pour la prévision et la gestion du risque pour les skieurs.
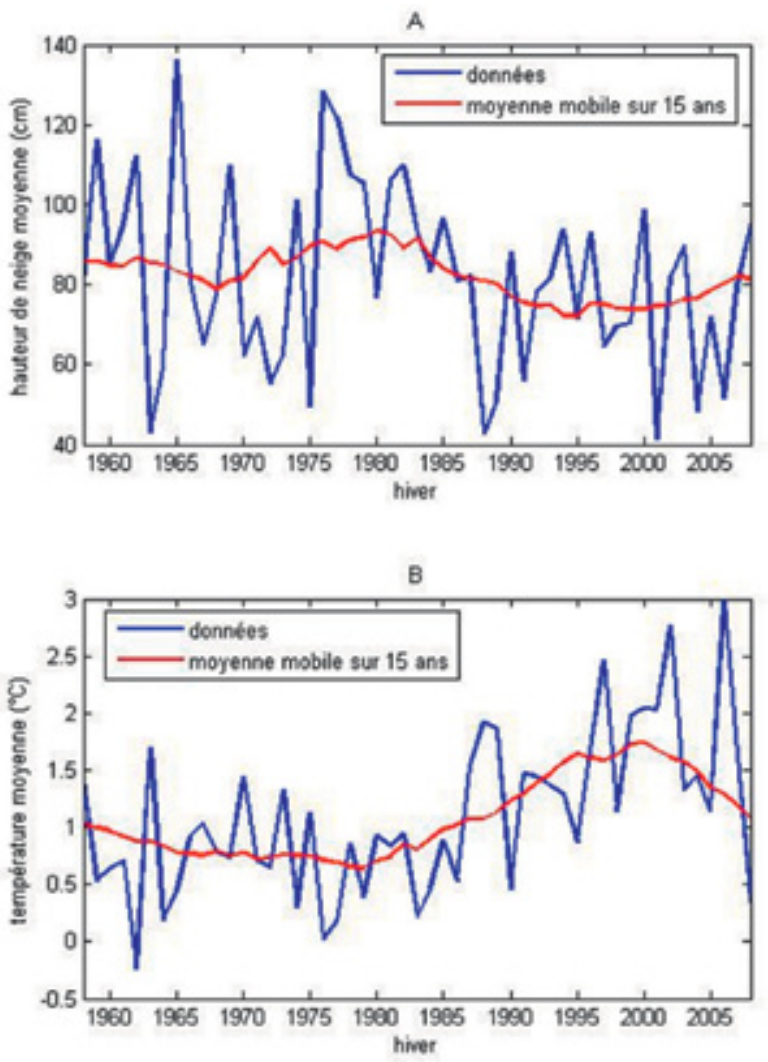

Figure 2 : Hauteur de neige et température hivernale à $2400 \mathrm{~m}$ dans les Alpes françaises: moyenne sur les 23 massifs d'après Durand et al., [2009a\&b]. 
Quelques résultats de recherches récentes fournissent des éclairages intéressants. Des données paléo-environnementales documentent un certain lien, à l'échelle séculaire, entre activité avalancheuse et évolution du climat [Corona et al., 2010]. En ce qui concerne les données d'observation d'avalanches, la France dispose de l'Enquête Permanente sur les Avalanches, véritable observatoire alimenté par les agents de l'Office National des Forêts (ONF) depuis le tout début du XX $X^{\mathrm{e}}$ siècle [Mougin, 1922] et géré par Irstea depuis 1973 avec le soutien financier du Ministère de l'Environnement depuis 2002. L'analyse de ces observations s'est pour l'instant concentrée sur la période post seconde guerre mondiale où les observations sont plus homogènes. Des méthodes statistiques avancées adaptées [Eckert et al., 2010a\&b] ne montrent pas d'évolution monotone. Néanmoins, malgré une forte variabilité interannuelle, un maximum d'activité autour de 1980 a pu être identifié dans les nombres d'avalanches, suivi par une décroissance (Figure 3A). Ce motif se retrouve, de manière amplifiée et inversée, dans les altitudes d'arrêt atteintes par les avalanches (Figure 3B), et plus encore dans les altitudes d'arrêt atteintes par les grandes avalanches importantes pour le zonage, qui augmentent depuis 1980 (Figure 3C). Une autre tendance qui se dégage de manière claire est la diminution régulière de la proportion des avalanches avec aérosols depuis le milieu des années soixante-dix, date à partir de laquelle cette information est enregistrée dans l'EPA (Figure 3D).

L'augmentation relative de l'activité avalancheuse sur la période 1960-1980 correspond bien à des hivers plus rigoureux, par ailleurs bien documentés par d'autres indicateurs climatiques (courtes avancées glaciaires notamment que des méthodes d'analyse similaires permettent de mettre en évidence [Thibert et al., 2013]). Le " recul des avalanches» ensuite observé, accompagné d'une diminution de la proportion des avalanches avec aérosol s'inscrit quant à lui dans une période de réchauffement marqué. Enfin, depuis quelques années (2000), les hivers ont à nouveau été plus rigoureux, avec davantage d'avalanches d'intensité importante (Figure 3).

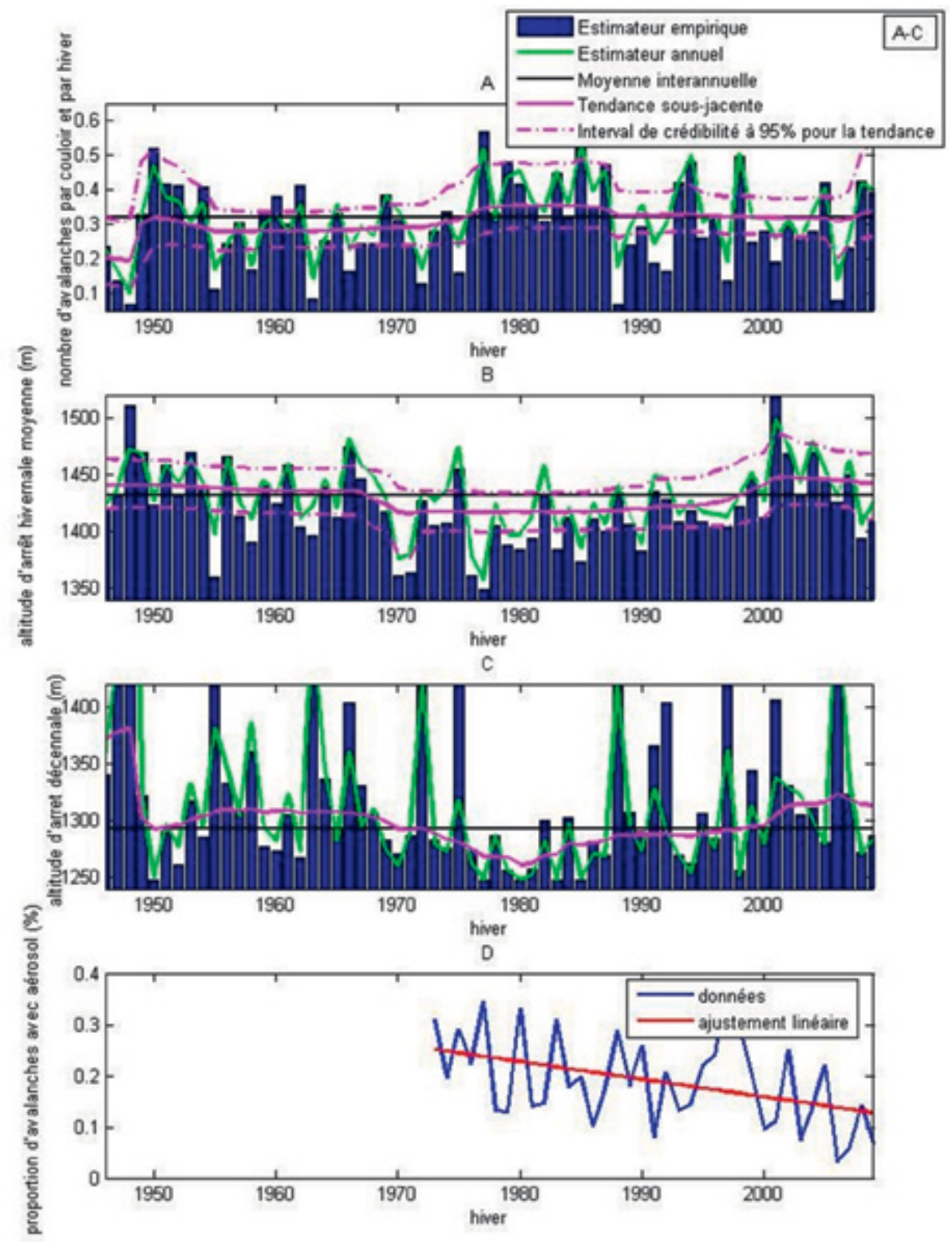

Figure 3 : Evolutions récentes dans l'activité avalancheuse dans les Alpes françaises, d'après Eckert et al. [2013]. A) Nombre d'avalanches par couloir et par an ; B) Altitude d'arrêt moyenne annuelle ; C) Altitude d'arrêt décennale (niveau de retour) ; D) Proportion d'avalanches avec aérosol (incluant les écoulements mixtes). 
Ce schéma global masque en réalité des évolutions différenciées contrôlées par l'altitude [Lavigne et al., 2012 ; 2015]. A basse altitude, la réduction d'activité depuis 1980 environ a été drastique, en lien avec la forte réduction de l'enneigement induite par le réchauffement climatique. Au contraire, à haute altitude, l'activité a récemment augmenté, peut-être sous l'effet de l'augmentation de la variabilité climatique hivernale précédemment mentionnée. Ainsi, de manière globale, les évolutions entre activité avalancheuse et climat hivernal dans les Alpes françaises apparaissent cohérentes.

Le lien entre altitudes d'arrêt des avalanches et température a pu être mis en évidence de manière plus explicite dans [Naaim et al., 2013] au travers de corrélations entre les coefficients de frottement qui contrôlent la dynamique des avalanches et les propriétés de la neige. 730 avalanches de 26 couloirs bien documentés de la haute vallée de l'Arve ont été rétro analysées par modélisation numérique. Les conditions initiales et les propriétés de la neige sont issues des réanalyses réanalyses nivo-météorologique [Durand et al. 2009a] et les altitudes de départ et d'arrêt sont issues de l'EPA. L'un des principaux résultats est que la moyenne par classe des coefficients de frottement statique (i.e. coefficient de frottement à vitesse nulle, au sens de la loi de Voellmy) de la neige augmente quasi linéairement avec la température (Figure 4). Ce résultat confirme que l'augmentation des altitudes d'arrêt observées depuis 1980 peut clairement être attribuée à l'augmentation de la température.

L'ensemble de ces résultats et observations (augmentation de la température du manteau neigeux, liens statistiques très nets entre l'activité avalancheuse et un large ensemble de variables nivo climatiques à différentes échelles de temps et d'espace [Castebrunet et al., 2012], etc.) suggèrent aussi une évolution progressive de l'activité avalancheuse vers davantage d'écoulements mobilisant de la neige humide, tout au moins en proportion. Par la suite, nous nous efforcerons de l'établir formellement puis en discuterons les conséquences physiques et opérationnelles.

\section{EVOLUTION DES AVALANCHES HUMIDES DANS LE MASSIF DU MONT-BLANC}

L'augmentation de l'activité avalancheuse humide en hiver au cours des dernières décennies commence à être documentée [Pielmeir et al., 2013, Mitterer et Schweizer 2013]. Pour la haute vallée de l'Arve, la série temporelle des occurrences des avalanches référencées dans l'EPA entre 1958 et 2009 sur un échantillon de 26 couloirs bien documentés a été étudiée en distinguant les avalanches humides des autres avalanches. Les avalanches sont considérées comme humides dès lors que la teneur en eau liquide moyenne du manteau neigeux du jour de l'avalanche dépasse $10 \mathrm{~kg} \cdot \mathrm{m}^{-3}$. La figure 5 où est reportée la proportion annuelle des avalanches de neige humide, montre une tendance relativement claire à l'augmentation de la proportion des avalanches humides. Cette augmentation est concomitante avec le réchauffement récent.

Parmi les résultats des rétro-analyses effectuées par [Naaim et al., 2013], le lien entre coefficient de frottement statique des avalanches et teneur en eau liquide moyenne ne montre aucune tendance monotone tant que la teneur en eau liquide moyenne est inférieure à $30 \mathrm{~kg} \cdot \mathrm{m}^{-3}$ (Figure 6). Au-delà de ce seuil, un effondrement drastique du coefficient de frottement statique est observé. En moyenne, les

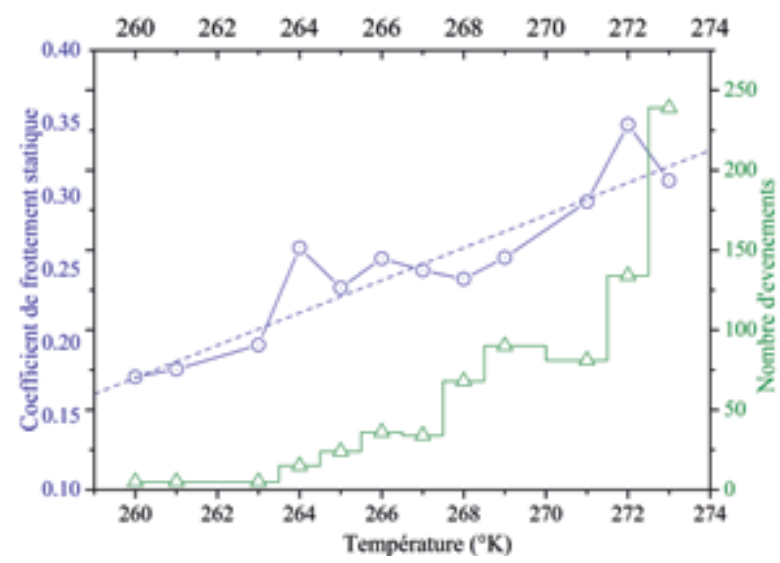

Figure 4 : Massif du Mont-Blanc : augmentation du frottement statique de la neige en écoulement avec la température moyenne de la neige, données issues de Naim et al. [2013] - cercle bleu plein : coefficient de frottement laxe de gauche et triangle vert plein : nombre d'évènements / axe de droite.

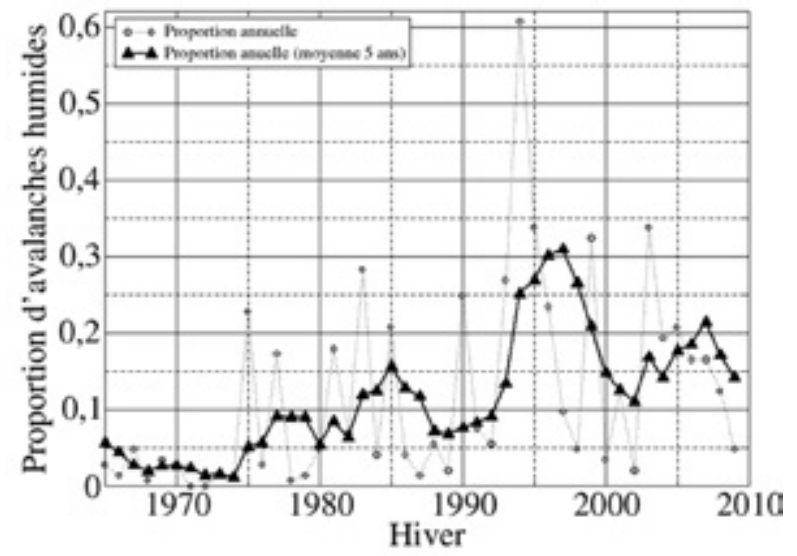

Figure 5 : Evolution de la proportion des avalanches humides des 26 couloirs étudiés (une avalanche est considérée comme humide si la moyenne de la teneur en eau liquide est supérieure à $10 \mathrm{~kg} . \mathrm{m}^{-3}$ )

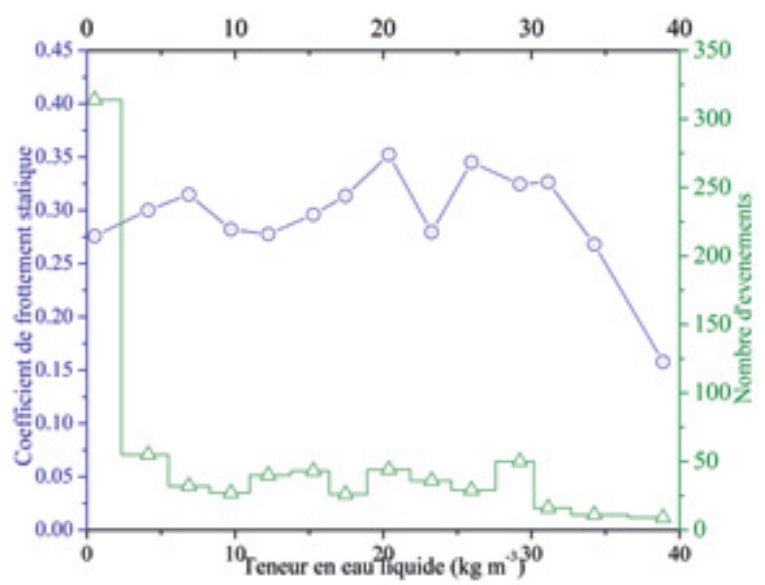

Figure 6 : Massif du Mont-Blanc : frottement statique de la neige en écoulement en fonction de la teneur en eau liquide, données issues de Naaim et al. [2013] - cercle bleu plein : coefficient de frottement / axe de gauche et triangle vert plein : nombre d'évènements / axe de droite. 
avalanches mobilisant de la neige dont la teneur en eau liquide est supérieure à $30 \mathrm{~kg} \cdot \mathrm{m}^{-3}$ ont un coefficient de frottement statique du même niveau que celui de la neige sèche très froide. Ainsi, contrairement à l'augmentation moyenne des altitudes d'arrêt avec la température, attribuable à une neige de plus en plus « chaude », on peut s'attendre à des altitudes d'arrêt basses voire très basses lorsqu'il s'agit d'avalanches de neige très humide. C'est le cas de l'avalanche de Saint François Longchamp.

\section{CONNAISSANCES RÉCENTES SUR LA PRESSION D'IMPACT ET IMPLICATIONS POUR LES AVALANCHES HUMIDES}

La pression d'impact des avalanches sur une structure est la force que subit la structure par unité de surface lors de son interaction avec l'écoulement avalancheux. Par analogie avec les écoulements turbulents pleinement développés, la pression d'impact a été longtemps calculée comme le produit de la pression dynamique de l'écoulement libre, c'est à dire l'énergie cinétique par unité de volume, et d'un coefficient de trainée qui ne dépend que de la forme de l'obstacle. Dans l'ingénierie paravalanche, les valeurs recommandées pour ce dernier sont dans l'intervalle 0.5 à 2 .

De nombreux travaux expérimentaux, conduits récemment sur des sites test [Sovilla et al., 2008 ; 2010 ; Thibert et al., 2008 ; Baroudi et Thibert, 2009 ], ont montré un comportement singulier en matière de pression d'impact des avalanches lentes et épaisses, classe à laquelle appartiennent les avalanches de neige humide qui s'écoulent lentement avec des épaisseurs et des masses volumiques très élevées.

Nous résumons dans ce qui suit les connaissances obtenues pour d'une part les structures étroites dont la largeur est faible devant l'épaisseur de l'écoulement (pylônes) et d'autre part les structures de largeur grande devant l'épaisseur d'écoulement (murs).

\section{IV.1. Cas des obstacles étroits}

Dans le cas des structures de largeur relative faible par rapport à l'épaisseur de l'écoulement, les mesures obtenues sur les sites de terrain du Lautaret (Hautes Alpes, France) et de la Sionne (Valais, Suisse), équipés de mâts de mesures dotés de capteurs de vitesses, de pressions et d'épaisseurs, ont montré que le coefficient de trainée, rapport de la pression d'impact à la pression dynamique [Comolet, 1982], était une fonction décroissante du nombre de Froude [Sovilla et al., 2008 ; Thibert et al., 2008]. Les écoulements à surface libre étant considérés hydrostatiques, la contribution de la pression hydrostatique sur les capteurs de pression est retranchée à la pression d'impact.

Ce résultat rappelle les écoulements des fluides visqueux à très faibles nombres de Reynolds ( $\mathrm{Re})$, dit écoulements rampants, dans lesquels le coefficient de trainée dépend de Re et peut être approché par la solution théorique $(\mathrm{Cd}=64 / \mathrm{Re})$. Pour les écoulements à très grand nombre de Reynolds, le coefficient de trainée est quasi constant $(\mathrm{Cd}=0.3$ à 2$)$ et ne dépend que de la forme de la structure impactée. Pour tout écoulement à surface libre d'un fluide quelconque, on peut définir une viscosité cinématique effective (dépendante des propriétés du fluide et des grandeurs de l'écoulement) en divisant la contrainte tangentielle par le produit du taux de cisaillement et de la masse volumique. C'est ce que nous avons fait pour les mesures obtenues sur les sites du Lautaret et de la Sionne. Pour chaque mesure de $\mathrm{Cd}$, la connaissance de l'épaisseur $\mathrm{H}$, de la vitesse moyenne $\mathrm{V}$ et de la pente locale du terrain $\theta$, nous a permis de déterminer la viscosité cinématique effective d'un fluide Newtonien équivalent qui s'écoulerait en régime permanent uniforme sur la même pente avec la même vitesse et la même épaisseur. A partir de la viscosité ainsi obtenue, de la vitesse et du diamètre de l'obstacle, nous avons construit un nombre de Reynolds équivalent $R_{e m}=\frac{3 F^{2}}{\tan \theta} \frac{d}{H}$ (où $F=\frac{V}{\sqrt{g H}}$ est le nombre de Froude et $d$ la largeur de l'obstacle [Naaim et al., 2008]).

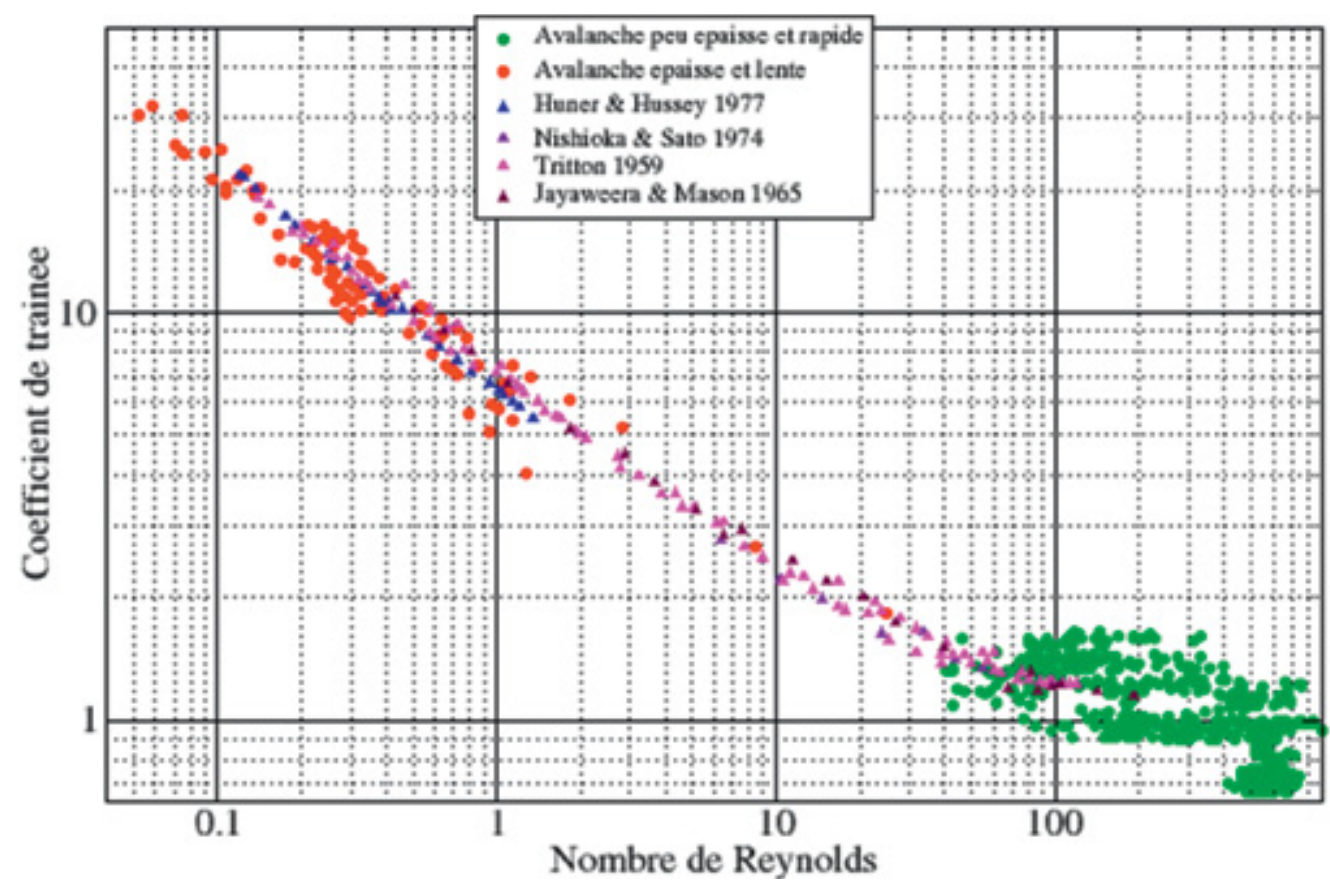

Figure 7: Comparaison du coefficient de trainée entre les fluides visqueux (données extraites de [Tritton, 1959 ; Huner et Hussey, 1977 ; Jayaweera et Mason, 1965 ; Nishioka et Sato, 1974]), les avalanches rapides et les avalanches lentes 
Cette analyse a été appliquée à deux avalanches denses très différentes [Sovilla et al., 2008, Thibert et al., 2008]. Sur le même graphe nous avons reporté les résultats des deux avalanches et les données issues de la littérature relatives aux coefficients de trainée des fluides Newtoniens à nombres de Reynolds modérés [Tritton, 1959 ; Huner et Hussey, 1977 ; Jayaweera et Mason, 1965 ; Nishioka et Sato, 1974]. Dans cette gamme, le coefficient de trainée décroit fortement avec le nombre de Reynolds. Les données des avalanches et des fluides Newtoniens suivent les mêmes tendances. Il ressort que les avalanches lentes et épaisses s'écoulent en régime de faible nombre de Reynolds et que leur coefficient de trainée dépend du nombre de Reynolds de manière très forte. Il peut ainsi atteindre 30 pour des nombres de Reynolds voisins de 0.1 , notamment tout près de l'arrêt. Ces avalanches doivent donc être traitées avec beaucoup d'attention quant à leur pression d'impact. Les avalanches rapides et peu épaisses s'écoulent quant à elles à des nombres de Reynolds élevés et ont un coefficient de trainée faible de l'ordre de l'unité.

\section{IV.2. Cas des obstacles larges}

Dans le cas des structures de dimension voisine de l'épaisseur de l'écoulement, qui modifient donc largement l'écoulement (forte déviation des lignes de courant internes et à la surface libre de l'écoulement), l'approche basée sur un coefficient de trainée n'est théoriquement plus valide. Des effets spécifiques en lien avec la forte modification de la géométrie de l'écoulement au voisinage de l'obstacle doivent être pris en compte.

Par exemple, une équation générale donnant la force subie par un mur soumis à un écoulement gravitaire à surface libre a été récemment proposée et validée pour les écoulements denses granulaires [Faug et al., 2009, 2012 ; Chanut et al., 2010] et en partie confrontée à des mesures de terrain pour le cas de la neige [Faug et al., 2010]. Cette équation prend en compte la formation d'une zone quasi-statique, dite « zone morte ", qui coexiste avec une zone inertielle au-dessus capable de déborder le mur. Un bilan de masse et de quantité de mouvement dans le volume $\mathrm{V}_{0}$ perturbé par la présence du mur (zone morte et zone inertielle au-dessus) montre que la force est alors principalement exprimée comme la somme de trois contributions : (i) une première contribution liée à la variation d'énergie cinétique dans le volume $V_{0}$ (proportionnelle au carré de la vitesse de l'écoulement incident), (ii) une seconde contribution liée à la pression hydrostatique de l'écoulement incident (proportionnelle à la hauteur de l'écoulement incident), et (iii) une troisième contribution liée au poids apparent dans la direction de l'écoulement du volume $V_{0}$. Cette dernière contribution résulte du poids $d u$ volume $\mathrm{V}_{0}$ diminué de la force de frottement et est dominante en régime d'écoulement lent et épais, ce qui est généralement le cas pour des avalanches de neige humide.

Une formule empirique a été proposée pour estimer la longueur de la zone morte et la contribution à la force totale qui en résulte [Faug et al., 2009 ; 2011]. Cette contribution associée à la présence de la zone morte augmente quand la dimension de l'obstacle augmente, et dépend aussi de la masse volumique du matériau. Elle peut s'accroitre fortement quand la masse volumique de la zone morte augmente par compaction, et cet effet peut probablement être amplifié par une augmentation de la teneur en eau liquide de la neige. Elle dépend aussi du frottement de la neige. Comme pour le cas des obstacles étroits, il faut donc prêter une attention toute particulière au cas des avalanches de neige humide lorsqu'il s'agit de calculer leur pression d'impact sur les ouvrages dans les zones d'arrêt des avalanches.

\section{EVOLUTION FUTURE PRÉVISIBLE DU MANTEAU NEIGEUX ET DE L'ACTIVITE AVALANCHEUSE}

Pour ce qui est des prédictions de l'activité avalancheuse future, la question est complexe [IPCC, 2012]. La communauté des climatologues dispose de scénarii climatiques globaux décrivant les évolutions à l'échelle de quelques centaines de kilomètres carrés mais ne pouvant représenter ni les phénomènes météorologiques locaux ni l'enneigement. Avant de passer à l'activité avalancheuse, il est donc nécessaire de passer par des projections climatiques à la résolution des massifs montagneux puis d'utiliser en complément un modèle de simulation pour le manteau neigeux. Ainsi, l'évolution de nombreux paramètres nivo-météorologiques (couverture neigeuse, températures minimale et maximale diurnes, précipitations pluvieuses et neigeuse, etc.) a-t-elle pu être quantifiée à l'échelle des massifs montagneux français (Mont-Blanc, Vanoise, Mercantour, etc.) et déclinée en fonction de l'altitude (pas de $300 \mathrm{~m}$ ), l'exposition (Nord, Est, Sud, Ouest) et la pente (plat, 20 et 40 degrés). En outre, aucune projection climatique ne pouvant prétendre décrire à elle seule le futur, différents modèles climatiques ont été utilisés pour élaborer un éventail de scénarios, leur comparaison permettant d'évaluer les marges d'incertitude des résultats. Du fait de la lourdeur de la chaîne de modélisation nécessaire, ce sont pour l'instant seulement les résultats de l'avant dernier rapport du GIEC qui ont été utilisés [IPCC, 2007], plutôt que les derniers scenarii, nettement affinés [IPCC, 2013].

Selon les paramètres climatologiques considérés, les résultats issus des divers modèles/scenarii sont très proches (températures, très directement liées aux scénarios d'émission de gaz à effet de serre) ou plus hétérogènes (précipitations, dont la représentation peut varier d'un modèle à l'autre [Rousselot et al., 2012]). Pour résumer, le réchauffement attendu (valeur moyenne de $+1,5{ }^{\circ} \mathrm{C}$ en milieu de siècle et de +2 à $+4{ }^{\circ} \mathrm{C}$ en fin de siècle), et des précipitations relativement stationnaires contraindront fortement, en interaction avec la topographie, l'évolution des variables liées à la neige. En particulier, une réduction drastique du manteau neigeux sec est bel et bien à prévoir à toutes les échelles spatio-temporelles considérées. De même, une augmentation des quantités de neige humide est prévisible, en tout cas à haute altitude et/ou en " plein hiver », i.e. là où elle est pour l'instant peu présente. À toutes les altitudes, les conditions de neige humide devraient apparaître plus tôt dans la saison. A basses altitudes, le réchauffement sera toutefois suffisamment fort pour réduire même le manteau humide. Globalement, par rapport à la période de référence, les changements sont certes très forts pour la fin du $\mathrm{XXI}^{\mathrm{e}}$ siècle, mais déjà très importants dès le milieu du siècle. Les changements en hiver seront moins importants qu'au printemps. A la même altitude, le sud des Alpes françaises ne sera pas beaucoup plus touché que le nord des Alpes Françaises, ce qui signifie que le manteau neigeux sera plus préservé dans les massifs du sud qui ont une altitude moyenne plus élevée (Figure 8, A-B).

En ce qui concerne l'activité avalancheuse, la combinaison de ces résultats avec les relations statistiques établies par Castebrunet et al., [2012] conduit à prédire une diminution globale du nombre d'avalanches de 20 à $30 \%$ (Figure 8C) pour le $\mathrm{XXI}^{\mathrm{e}}$ siècle [Castebrunet et al., 2014]. La baisse sera bien plus drastique au printemps et à basse altitude du fait d'une quasi-disparition du manteau neigeux (Figure 8E). En revanche, tant que le manteau neigeux restera substantiel, on s'attend à une augmentation de l'activité avalancheuse 


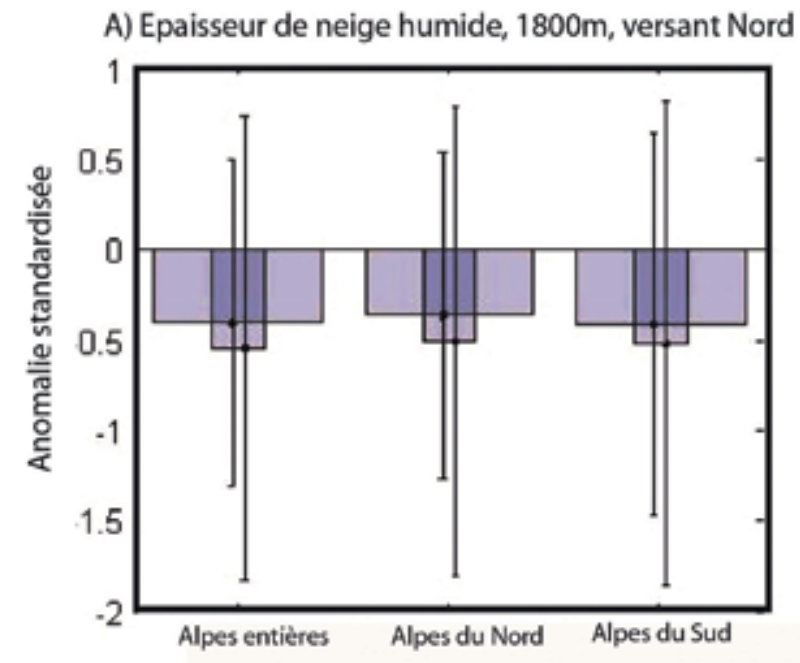

B) Epaisseur de neige humide, $3000 \mathrm{~m}$, versant Nord

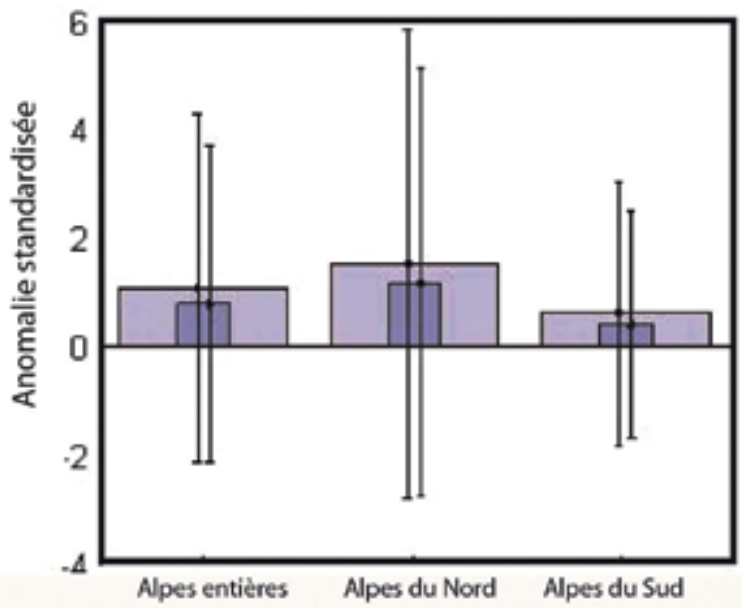

C) Activité avalancheuse, hiver entier (15 décembre-15 juin)

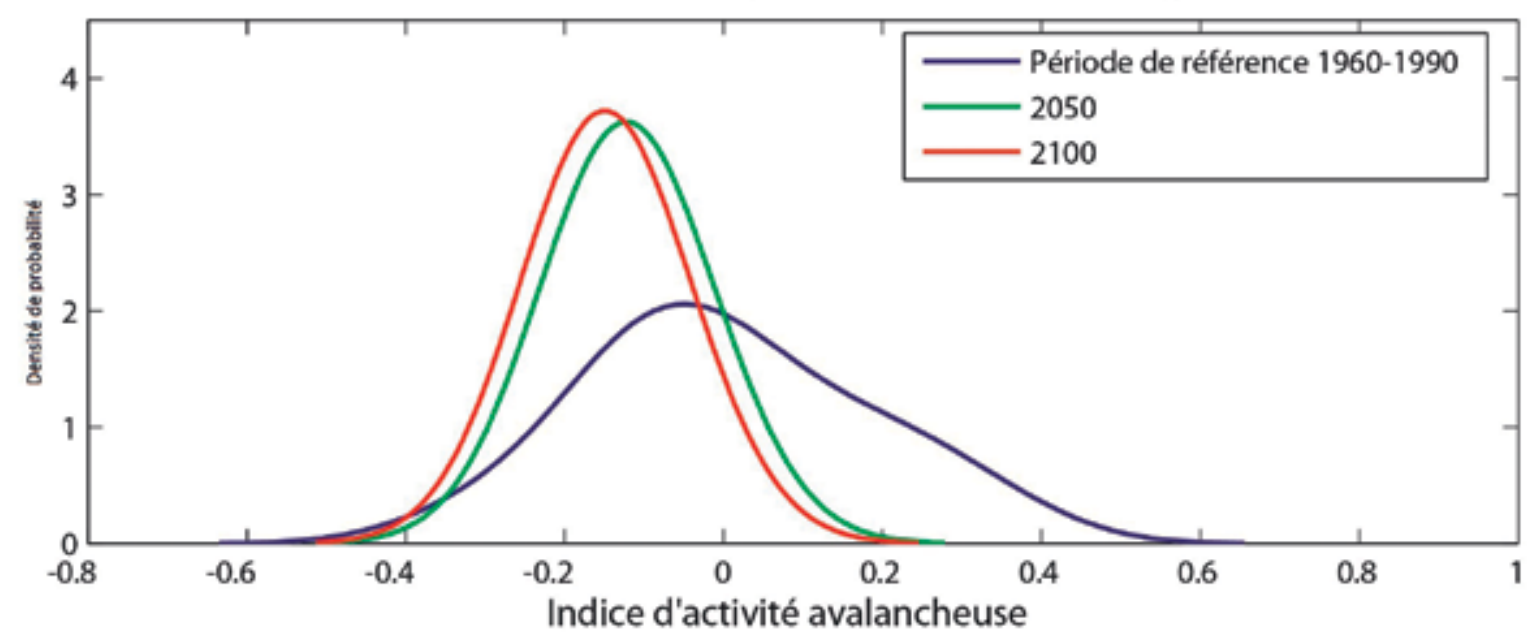

D) Activité avalancheuse, 15 décembre - 15 mars

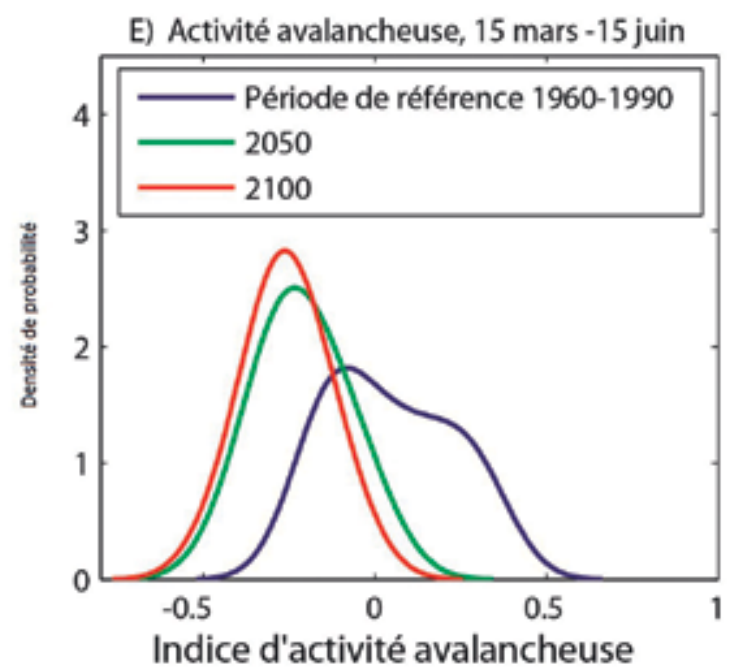

Figure 8 : Projections futures, anomalie standardisée par rapport à la période de référence 1960-1990 d'après [Castebrunet et al., 2014]. Évolution des épaisseurs humides en versant nord à A) $1800 \mathrm{~m}$ et B) $3000 \mathrm{~m}$. Barre très épaisse pour le milieu et barre épaisse pour la fin du XXIème siècle. Les traits fins représentent la variabilité interannuelle associée. Distribution interannuelle d'un indice d'activité avalancheuse totale standardisé [Castebrunet et al., 2012]: C) Hiver entier ; D) Plein hiver ; E) Printemps. 
en plein hiver à haute altitude (Figure 8D). Pour l'obtention de ces résultats, la distinction avalanche de neige sèche / avalanche de neige humide n'a pas été explicitement faite, et aucune ségrégation altitudinale des couloirs n'a été utilisée. Ces résultats s'expliquent néanmoins assez naturellement par des conditions favorables aux avalanches de neige humide plus tôt dans la saison, ce qui « boucle la boucle » avec l'augmentation de la proportion d'avalanches de neige humide par rapport aux avalanches de neige sèche déjà prédite par Martin et al. [2001] sur la base de scenarii d'évolution climatique bien plus rustiques.

\section{CONCLUSIONS ET PERSPECTIVES}

Lors des récents hivers, les Alpes françaises, ont connu plusieurs épisodes de forte activité d'avalanches de neige humide. Ces avalanches ont parcouru des distances exceptionnelles et ont provoqué des dégâts importants y compris là où leurs vitesses étaient faibles. La répétition de ces évènements nous a conduit à explorer les liens entre divers indicateurs de l'activité avalancheuse et le réchauffement climatique. Le croisement de l'Enquête Permanente sur les Avalanches et les réanalyses nivo-météorologique, a montré que depuis la fin des années 1970 des changements importants ont été constatés. Les fréquences moyennes ont diminué, les altitudes d'arrêt ont augmenté et la proportion des avalanches avec aérosols a diminué. Ces évolutions sont concomitantes avec l'augmentation de la température moyenne dans les Alpes sur la même période. Les deux mêmes bases de données ont été exploitées pour étudier, sur la haute vallée de l'Arve, les corrélations entre les propriétés de frottement de la neige en mouvement et les propriétés de la neige mobilisée. La rétro analyse de 730 avalanches par modélisation numérique a permis de mettre en évidence trois tendances très importantes pour la dynamique des avalanches. La première tendance concerne la proportion des avalanches humides qui a augmenté depuis le milieu des années 1970. Le second résultat concerne l'augmentation importante du coefficient de frottement de la neige en mouvement avec la température moyenne. Plus la température moyenne de la neige est importante plus le frottement est important et l'altitude d'arrêt résultante plus haute. Ce résultat est parfaitement cohérent avec les tendances observées quant à l'évolution des altitudes d'arrêt depuis 1980. La troisième tendance est la forte diminution du coefficient de frottement dynamique dès que la teneur moyenne en eau liquide dépasse le seuil de $30 \mathrm{~kg} \cdot \mathrm{m}^{-3}$. Au-delà de ce seuil, un accroissement important des distances d'arrêt des avalanches est observé. Ce résultat confirme les tendances récentes observées pour les avalanches très humides.

Un ensemble de faits concordants tend à montrer que, du fait de l'augmentation de la température dans les Alpes depuis 1980, la proportion d'avalanches de neige humide augmente. Les projections climatiques pour le siècle à venir indiquent que cette tendance va se poursuivre même si globalement l'activité avalancheuse tendra à se réduire, sauf, sans doute, à haute altitude et en plein hiver.

En termes de risques, l'augmentation de l'humidité de la neige peut conduire à des avalanches dangereuses pour deux raisons : une augmentation des distances parcourues du fait d'un coefficient de frottement dynamique seuil faible, et des pressions d'impact nettement plus élevées que celles prédites pour des écoulements dominés par l'inertie. La connaissance actuelle du comportement mécanique de la neige humide est trop limitée pour quantifier précisément ces tendances.
Un effort de recherche doit donc à présent être porté sur la rhéologie de la neige humide en régime quasi-statique (déclenchement) et dynamique (propagation), et sur une analyse statistique spatio-temporelle à plus grande échelle explicitement dédiée aux avalanches de neige humide.

\section{REMERCIEMENTS}

Les auteurs remercient pour leurs soutiens la Direction Générale de la Prévention des Risques (http://www.avalanches.fr/), le Labex OSUG@2020 (Investissements d'Avenir, ANR-10-LABX-0056), le labex TEC21 (Investissements d'Avenir, ANR-11-LABX-0030) et le fond FEDER.

\section{REFERENCES}

Baroudi D., And ThiBerT E. (2009) - An instrumented structure to measure avalanche impact pressure: error analysis from Monte Carlo Simulations. Cold Reg. Sci. Tech. 59(2-3), 242-250.

Beniston M. (2005) - Warm winter spells in the Swiss Alps: Strong heat waves in a cold season? A study focusing on climate observations at the Saentis high mountain site. Geophys. Res. Lett. 32, L 01812.

Castebrunet H., Eckert N., Giraud G. (2012) - Snow and weather climatic control on snow avalanche occurrence fluctuations over $50 \mathrm{yr}$ in the French Alps. Climate of the Past. 8, 855-875.

Castebrunet H., Eckert N., Giraud G., Durand Y., Morin S. (2014) - Projected changes of snow conditions and avalanche activity in a warming climate: a case study in the French Alps over the 2020-2050 and 2070-2100 period. The Cryosphere. 8, 1673-1697.

Corona C., Rovera G., Lopez Saez J., Stoffel M., Perfettini P. (2010) - Spatio-temporal reconstruction of snow avalanche activity using tree rings: Pierres Jean Jeanne avalanche talus, Massif de l'Oisans, France. Catena. 83(2-3), 107-118.

Chanut B., Faug T., NaAim M. (2010) - Time-varying force from dense granular avalanches on a wall. Phys. Rev. E. 82, 041302.

Comolet R. (1982) - Dynamique Des Fluides Reels, Turbomachines. Mecanique Experimentale Des Fluides, Edition Masson. II.

Durand Y., Laternser M., Giraud G., Etchevers P., Lesaffre L., MERINDOL L. (2009) - Reanalysis of 44 year of climate in the French Alps (1958-2002): methodology, model validation, climatology, and trends for air temperature and precipitation. Journal of Applied Meteorology and Climatology. 48, Issue 3, 429-449.

Durand Y., Laternser M., Giraud G., Etchevers P., Merindol L., Lesaffre B. (2009) - Reanalysis of 47 Years of Climate in the French Alps (1958-2005): Climatology and Trends for Snow Cover. Journal of Applied Meteorology and Climatology. 48(12), 2487-2512.

ECKERT N. (2011) - Synthèse des travaux exploitant l'EPA pour l'étude des fluctuations climatiques en zone de montagne (Alpes Françaises). Note de synthèse à la DGPR du ministère de l'environnement. Convention MEEDDAT-ONF-Cemagref.21p.

Eckert N., Parent E., Kies R., BAyA H. (2010) - A spatio-temporal modelling framework for assessing the fluctuations of avalanche occurrence resulting from climate change: application to 60 years of data in the northern French Alps. Climatic Change. 101(3-4), 515-553.

Eckert N., BAyA H., Deschâtres M. (2010) — Assessing the response of snow avalanche runout altitudes to climate fluctuations using hierarchical modeling: application to 61 winters of data in France. Journal of Climate. 23, 3157-3180. 
Eckert N., Keylock C. J., Castebrunet H., Lavigne. A., NaAim M. (2013) - Temporal trends in avalanche activity in the French Alps and subregions: from occurrences and runout altitudes to unsteady return periods. J. Glaciol. 59(213), 93-114.

Faug T., Beguin R., Chanut B. (2009) - Mean steady granular force on a wall overflowed by free-surface gravity-driven dense flows. Phys. Rev. E. 80, 021305.

Faug T., Chanut B., Beguin R., NaAim M., Thibert M., Baroudi D. (2010) - A simple analytical model for pressure on obstacles induced by snow avalanches. Ann. Glaciol. 51 (54), 1-8.

Faug T., Caccamo P., Chanut B. (2011) - Equation for the force experienced by a wall overflowed by a granular avalanche: experimental verification. Phys. Rev. E. 84, 051301.

Faug T., Caccamo P., Chanut B. (2012) - A scaling law for impact force of a granular avalanche flowing past a wall. Geophys. Res. Lett. 39, L23401.

Huner B. And Hussey R.G. (1977) - Cylinder drag at low Reynolds number. Phys. Fluids. 20, 1211-1218.

IPCC (2007) - Climate change 2007: The physical science basis, in: Contribution of working group I to the fourth assessment report of the Intergovernmental Panel on Climate Change, edited by: Solomon, S., Qin, D., Manning, M., Chen, Z., Marquis, M., Averyt, K. B., Tignor, M., and Miller H. L., Cambridge University Press, Cambridge, UK and New York, NY, USA.2007.

IPCC (2012) - Managing the Risks of Extreme Events and Disasters to Advance Climate Change Adaptation. A Special Report of Working Groups I and II of the Intergovernmental Panel on Climate Change [Field, C.B., V. Barros, T.F. Stocker, D. Qin, D.J. Dokken, K.L. Ebi, M.D. Mastrandrea, K.J. Mach, G.-K. Plattner, S.K. Allen, M. Tignor, and P.M. Midgley (eds.)]. Cambridge University Press, Cambridge, UK, and New York, NY, USA.582 pp.

IPCC (2013) - Climate change 2013: The physical science basis. Contribution of Working Group I to the Fifth Assessment Report of the Intergovernmental Panel on Climate Change [Stocker, T.F., D. Qin, G.-K. Plattner, M. Tignor, S.K. Allen, J. Boschung, A. Nauels, Y. Xia, V. Bex and P.M. Midgley (eds.)]. Cambridge University Press, Cambridge, United Kingdom and New York, NY, USA.1535 pp.

JayAweERA K., AND B. J. MASON (1965) - The behavior of freely falling cylinders and cones in a viscous fluid. J. Fluid Mech 22, 709-720.

Lavigne A., Bel L., Parent E., Eckert N. (2012) - A model for spatio-temporal clustering using multinomial probit regression: application to avalanche counts in the French Alps. Envirometrics. 23, 522, 534.

Lavigne A., Eckert N., Bel L., Parent E. (In Press). Adding Expert Contribution To The Spatio-Temporal Modeling Of Avalanche ACtivity Under DifFerent Climatic Influences. Journal of the Royal Statistical Society C. (Applied Statistics), in press.
LAzar B., Williams M. (2008) - Climate change in western ski areas: Potential changes in the timing of wet avalanches and snow quality for the Aspen ski area in the years 2030 and 2100, Cold Regions Science and Technology 51. 219-228.

Martin E., Giraud G., Lejeune Y., Boudart G. (2001) - Impact of climate change on avalanche hazard. Ann. Glaciol. 32, 163-167.

Mougin P. (1922) — Les avalanches en Savoie. Ministère de l'Agriculture, Direction Générale des Eaux et Forêts, Service des Grandes Forces Hydrauliques, Paris. 175-317.

NaAim M., Faug T., Thibert M., Eckert N., Chambon G., NaAim F., Bellot H. (2008) - Snow avalanches pressure on obstacles. Proceedings of the International Snow Science Workshop 2008, 21-27 September 2008, Whistler, BC, Canada, International Snow Science Workshop Canada Inc. (eds).740-746.

NaAim M., Durand Y., Eckert N., Chambon G. (2013) - Dense avalanche friction coefficients : influence of physical properties of snow. Journal of Glaciology. 59(216), 771-782.

Nishioka M., AND H. SATO. (1974) - Measurements of velocity distributions in the wake of a circular cylinder at low Reynolds numbers. J. Fluid Mech. 65, 97-112.

Pielmeier C., Techel F., Marty C., Stucki T. (2013) - Wet snow avalanche activity in the Swiss Alps trend analysis for mid-winter season. proceedings of International Snow Science Workshop Grenoble - Chamonix Mont-Blanc.2013.

Sovilla B., Schaer M., Kern M., And Bartelt P. (2008) Impact pressures and flow regimes in dense snow avalanches observed at the vallée de la Sionne test site. J. Geophys. Res. 113, F01010.

Sovilla B., Kern M., And Schaer M. (2010) - Slow drag in wet-snow avalanche flow. J. Glaciol. 56(198), 587-592.

Thibert E., Baroudi D., Limam A., Berthet-Rambaud P. (2008) Avalanche impact pressure on an instrumented structure. Cold Reg. Sci. Tech. 54, 206-215.

Thibert E., EcKert N., Vincent C. (2013) - Climatic drivers of seasonal glacier mass balances: an analysis of 6 decades at Glacier de Sarennes (French Alps). The Cryosphere. 7(1), 47-66.

TRITTON D. J. (1959) - Experiments on the flow past a circular cylinder at low Reynolds numbers. J. Fluid Mech. 6, 547-567.

Mitterer C., Schweizer J. (2013) - Analysis of the snow-atmosphere energy balanche during wet snow instabilities and implications for avalanche prediction. The Cryosphere. 7, 205-216.

Vera Valero C., Wikstroem Jones K., BüHler Y., Bartelt P. (2015) - Release Temperature, Snow cover Entrainment and the Thermal Flow Regime of Snow Avalanches. Journal of Glaciology. 61(225), 173-184. 